\title{
Intranasal atomised dexmedetomidine optimises surgical field visualisation with decreased blood loss during endoscopic sinus surgery: a randomized study*
}

\author{
H. Qiao, J. Chen, W. Li, X Shen
}

Department of Anesthesiology, The Eye Ear Nose and Throat Hospital, Fudan University, Shanghai, China
Rhinology 54: 38-44, 2016

DOl:10.4193/Rhino15.085

*Received for publication:

March 30, 2015

Accepted: May 25, 2015

\begin{abstract}
Background: Safe and effective endoscopic sinus surgery (ESS) depends on distinct surgical visibility. Various interventions are proposed to reduce intranasal bleeding. This study investigated whether intranasal atomised dexmedetomidine (DEX) provided optimal surgical conditions and decreased blood loss.
\end{abstract}

Methods: ASA I or II patients undergoing ESS were randomly assigned to receive either $2 \mu \mathrm{g} / \mathrm{kg}$ intranasal DEX (group D) or the same volume of saline (group N) 15 min before induction. Lund-Mackay (LM) scores represented the extent of the preoperative surgical lesion and were obtained based on the computed tomographic scans. Estimated blood loss was recorded. The visibility of the surgical field was rated by surgeons on a numerical rating scale (NRS) or assessed using Boezaart score.

Results: Median blood loss in groups D and N was 75 and $100 \mathrm{ml}$, respectively. NRS and Boezaart score for surgical condition were lower in group D than in group N. LM score showed a positive correlation between NRS and Boezaart score in group N but not in group D.

Conclusion: Intranasal atomised DEX resulted in improved surgical conditions with less bleeding during ESS despite the severity of the preoperative surgical lesion.

Key words: anaesthesia, otolaryngology, anaesthetics, dexmedetomidine, blood loss

\section{Introduction}

During endoscopic sinus surgery (ESS), increased bleeding results in a poor display of the various structures and anatomical landmarks and increases the risk of potential complications, such as skull base injury, cerebrospinal fluid leakage, orbit penetration, and damage to the optic nerve and internal carotid artery ${ }^{(1,2)}$. Optimised visualisation of the anatomy would not only decrease risks and improve surgical outcomes, but may also reduce operative time and decrease blood loss ${ }^{(3)}$.

Therefore, numerous interventions to optimise surgical visibility have been investigated. Certain procedures, such as reverse Trendelenburg positioning, topical decongestants, infiltration of local anaesthetics, preoperative use of prednisone, heart rate control, and manipulation of general anaesthesia ${ }^{(4)}$, have been shown to reduce intraoperative blood loss and improve visuali- sation of the surgical field.

Intravenous or oral premedication with clonidine, an a-2 adrenoceptor agonist, effectively reduces bleeding ${ }^{(5)}$, shortens surgical times ${ }^{(6)}$, and provides better visualisation of the surgical field and a more favourable haemodynamic profile ${ }^{(7)}$ during ESS. Furthermore, peripheral or local administration of clonidine has also proven helpful in regional anaesthesia, neuraxial blockade, and intra-articular analgesia ${ }^{(8)}$. The mechanism of the peripheral effects of a-2 adrenoceptor agonists has not been fully elucidated but is suggested to be the result of local vasoconstriction (9) and/or direct inhibition of impulse conduction in peripheral nerves ${ }^{(10,11)}$.

As dexmedetomidine (DEX) has an approximate eight-fold a-2 to $a-1$ selectivity and is four to five times more potent than clonidine, its peripheral effects may be even more efficacious 
$(12,13)$. Intranasal administration of DEX for sedation and pain relief has been found to be effective, reliable, well tolerated, and convenient in healthy volunteers ${ }^{(14)}$ and patients undergoing third molar surgery ${ }^{(15,16)}$. To our knowledge, no clinical study has been conducted to investigate the local effects of intranasal DEX on intraoperative bleeding during ESS. We conducted this double-blind, randomized controlled study to assess the efficacy of intranasal atomised DEX in intraoperative field conditions during ESS under balanced anaesthesia.

\section{Methods}

The trial was approved by the institutional review board of the Eye and ENT hospital affiliated with Fudan University and registered with the Chinese Clinical Trial Registry (registration number ChiCTR-IOR-14005677). Written informed consent was obtained from each patient who enrolled in the study.

\section{Patients}

Patients with ASA grade I (a normal, otherwise healthy patient) or grade II (a patient with mild systemic diseases) undergoing ESS for chronic sinusitis under sevoflurane/remifentanil anaesthesia were consecutively recruited for the study. According to random, computer-generated allocation, patients were assigned to the following groups: intranasal premedication with DEX (group D) or intranasal placebo of normal saline (group N). The allocation sequence (contained in sequentially numbered, opaque, sealed and stapled envelopes) was concealed from the investigator enrolling and assessing participants. Patients with diseases or medications related to coagulation or the cardiovascular system were excluded.

The computed tomographic (CT) scans of all patients were reviewed before surgery by otorhinolaryngological surgeons. The Lund-Mackay (LM) CT score was obtained according to the degree of opacification of the involved sinus ( 0 , no opacification; 1, partial opacification; or 2, obstruction) and the degree of obstruction of the osteomeatal complexes ( 0 , no obstruction; 2 , obstruction), which represented the severity of sinus disease ${ }^{(17)}$. Patients with a total LM score greater than 12 were called highLM score patients and those with a total LM score of 12 or less were called low-LM score patients.

\section{Treatment and assessement}

Intranasal medications were prepared and administered by an anaesthesiologist who was not involved in the measurements or evaluations. In the preparation room, either $2 \mu \mathrm{g} / \mathrm{kg}$ DEX (group D) or the same volume of $0.9 \%$ saline (group N) was sprayed into both nostrils $15 \mathrm{~min}$ before induction. The syringe containing DEX or saline was attached via a lure lock connector to a nasal mucosal atomisation device (MADgic $\left.{ }^{\oplus}\right)$. Given that the concentration of the undiluted DEX solution was $100 \mu \mathrm{g} / \mathrm{ml}$ and both study drugs were clear solutions, the volume administered was identically $0.02 \mathrm{ml} / \mathrm{kg}$ in all groups to ensure blinding of the surgeon, the patients, and the investigators obtaining the data. Upon arrival in the operating theatre, noninvasive arterial blood pressure monitoring, electrocardiograms, and pulse oximetry were implemented for all patients. Anaesthesia was induced using intravenous propofol ( $2 \mathrm{mg} / \mathrm{kg})$, sulfentanyl $(0.2 \mu \mathrm{g} / \mathrm{kg})$, and rocuronium $(0.3 \mathrm{mg} / \mathrm{kg})$ in both groups. After insertion of a flexible laryngeal mask, sevoflurane was maintained at 0.8-1.2 minimum alveolar concentration (MAC) and a continuous remifentanil infusion was simultaneously initiated at a rate of $0.1-0.3$ $\mu \mathrm{g} / \mathrm{kg} / \mathrm{min}$ in both groups. The patients received mechanical ventilation in pressure-controlled mode with a tidal volume of $6 \mathrm{ml} / \mathrm{kg}$ at a frequency of 10 to $12 \mathrm{bpm}$ to provide end-tidal carbon dioxide concentration of 35 to $45 \mathrm{mmHg}$. Intravenous $0.5 \mathrm{mg} / \mathrm{kg}$ ketorolac tromethamine was given for postoperative analgesia.

The target mean blood pressure (MBP) was maintained at 55-65 $\mathrm{mmHg}$ by adjusting the sevoflurane concentration and remifentanil infusion rate within their ranges according to the anaesthesiologist's discretion. If this failed, a bolus of phenylephrine (40-80 $\mu \mathrm{g}$ ), ephedrine (3-6 mg), or esmolol (20-40 mg) was used for vasoactivity. An intravenous bolus of $0.3 \mathrm{mg}$ atropine was administered if the heart rate (HR) was less than $50 \mathrm{bpm}$. Two squeezed cotton balls soaked in epinephrine in a concentration of 1:80,000 was inserted into each nasal cavity. The surgical procedures were performed by three surgeons who had subspecialty training in rhinology and used a similar stepwise technique. All patients were positioned in 15 degrees reverse Trendelenburg for the entire procedure. The attending surgeons were unaware of the patient's premedication status.

Estimated blood loss was calculated by subtracting the total irrigation volume used for the procedure from the total amount of fluid in the suction canister at the end of surgery.

Immediately after surgery, the surgeons rated surgical visibility on a numerical rating scale (NRS), ranging from 0 to 10 , with 0 defined as the best condition and 10 as the worst ${ }^{(18)}$.

To make these assessments comparable to other ratings, the Boezaart grading scale was also used to rapidly evaluate intraoperative bleeding. Boezaart grading ranged from 0 to 5 as follows ${ }^{(19,20)}$ : 0 , no bleeding; 1 , slight bleeding: no suction of blood required; 2 , slight bleeding: occasional suctioning required, surgical field not threatened; 3 , slight bleeding: frequent suctioning required, bleeding threatens surgical field a few seconds after suctioning is removed; 4 , moderate bleeding, frequent suctioning required, bleeding threatens surgical field directly after suction is removed; 5 , severe bleeding: constant suctioning required, bleeding appears faster than can be removed by suction, surgical field severely threatened.

The anaesthesia time was defined as the time from anaesthesia induction to the end of all surgical manipulation and withdrawal of all operative instruments. Following surgery, the patients 


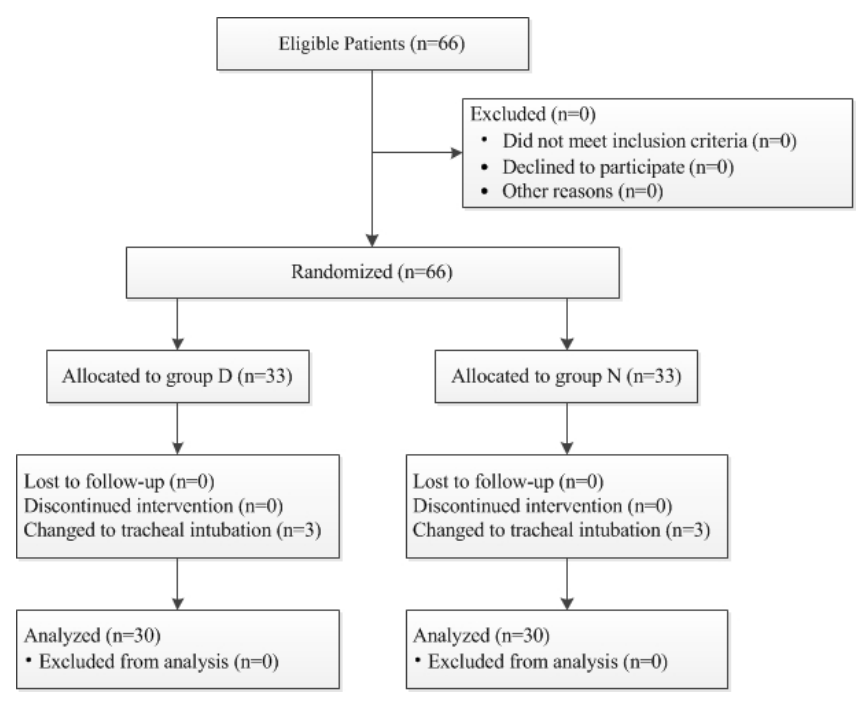

Figure 1. Consort diagram describing the flow of participants through each stage of a randomized trial.

were promptly transferred to the postanaesthesia care unit (PACU). An independent attending anaesthesiologist in the PACU was responsible for patient monitoring and management. The extubation time was defined as the time from the end of surgery to the time of extubation. Postoperative recovery was evaluated using a modified Alderet score and PACU time, defined as the time from the end of surgery to the time needed to achieve an Aldrete score greater than nine. Any event, such as coughing, hypotension, agitation, and hypoxemia, during emergence from anaesthesia was also recorded. Patients were also asked about recalling intraoperative events or any sign of awareness.

A research assistant who was blinded to the study and did not participate in patient care collected data, including postoperative pain rated by visual analogue scale (VAS), postoperative nausea and vomiting, sore throat, and other discomforts, $2 \mathrm{~h}$ after surgery.

\section{Statistical analysis}

The primary statistical endpoint was the NRS for surgical condition. Based on previous studies ${ }^{(21,22)}$, we calculated that 60 patients were required to give the study a statistical power of $80 \%$ at a $5 \%$ level of significance. Considering the probability of sample size loss, 10\% more patients were enrolled. The amount of blood loss, NRS for surgical condition, Boezaart score, average remifentanil infusion rate, average MAC, and VAS for pain intensity were described as the median (1 st/3rd quartiles) and were analysed using a Mann-Whitney rank sum test. Other continuous variables were expressed as the mean \pm SD and were analysed using the Student's t test. The categorical data were compared using the $\mathrm{x} 2$ test or the Fisher's exact test. Spearman's coefficients were used to describe the correlation of nonparametric data. All statistical assessments were two sided and considered significant if the P-value was less than 0.05 . Statistical analysis was performed with SPSS 15.0 software.

\section{Results}

Of the 66 patients enrolled in this study, data from 60 were sufficient for analysis (Figure 1). Three patients in each group switched to tracheal intubation following induction due to improper position of the laryngeal mask. Gender, age, weight, height, body mass index, ASA classification, total LM score, and the ratio of high-LM:low-LM score patients were similar in both groups (Table 1).

Pre-induction MBP and HR, duration of anaesthesia, average rate of the remifentanil infusion, average $M A C$, intraoperative fluid volume, and distribution of the three operators were also similar (Table 2). Intraoperative MBP was similar in both groups, whereas the average intraoperative HR was lower in group D. However, this difference was not statistically significant $(62 \pm 6$ vs. $66 \pm 11, \mathrm{P}=0.064$; Table 2).

The median (1st/3rd quartiles) blood loss was 100 (50/225) ml in group $\mathrm{N}$ and $75(0 / 188) \mathrm{ml}$ in group $\mathrm{D}(\mathrm{P}=0.029)$. The NRS of the surgical conditions was lower in group $D$ than in group $N[2$ $(1 / 3)$ vs. $3(2 / 5), P=0.007]$. Similarly, patients in group $D$ showed significant lower Boezaart scores [2 (1/2) vs. 2 (2/3), P = 0.001]. Extubation time was similar for both groups, but PACU time was longer in group $D$ than in group $N(35 \pm 8$ vs. $29 \pm 7, P=0.007$ ). The incidence of adverse events in the PACU and postoperative complications were similar in both groups (Table 3). NRS for po-

Table 1. Demographic characteristics.

\begin{tabular}{|lccc|}
\hline & $\begin{array}{c}\text { Group D } \\
(\mathbf{n}=\mathbf{3 0})\end{array}$ & $\begin{array}{c}\text { Group N (n } \\
\mathbf{=} \mathbf{3 0})\end{array}$ & P-value \\
\hline Sex (M/F) & $17 / 13$ & $19 / 11$ & 0.792 \\
\hline Age (years) & $52 \pm 12$ & $46 \pm 12$ & 0.076 \\
\hline Weight (kg) & $64.1 \pm 11.0$ & $65.0 \pm 12.1$ & 0.765 \\
\hline Height (cm) & $166.2 \pm 8.1$ & $165.7 \pm 7.7$ & 0.819 \\
\hline Body mass index (kg/m2) & $23.1 \pm 3.2$ & $23.6 \pm 3.4$ & 0.604 \\
\hline ASA classification (I/II) & $20 / 10$ & $26 / 4$ & 0.125 \\
\hline Total LM score & $8 \pm 6$ & $7 \pm 5$ & 0.656 \\
\hline Patients of high-:low-LM score & $6 / 24$ & $5 / 25$ & 1.000 \\
\hline
\end{tabular}

The values are presented as the mean \pm SD or the number of patients. Group D, dexmedetomidine group; Group N, 0.9\% saline group; LM, Lund-Mackay; High-LM score, LM score > 12; Low-LM score, LM score $\leq$ 12. 
Table 2. Perioperative variables.

\begin{tabular}{|c|c|c|c|}
\hline & $\begin{array}{l}\text { Group D } \\
(n=30)\end{array}$ & $\begin{array}{l}\text { Group N } \\
(n=30)\end{array}$ & P-value \\
\hline Pre-induction MBP $(\mathrm{mm} \mathrm{Hg})$ & $95 \pm 14$ & $100 \pm 14$ & 0.169 \\
\hline Pre-induction HR (beats/min) & $73 \pm 12$ & $78 \pm 11$ & 0.131 \\
\hline Operator $1 / 2 / 3$ & $7 / 9 / 14$ & $10 / 5 / 15$ & 0.426 \\
\hline $\begin{array}{l}\text { Average rate of remifentanil } \\
\text { infusion }(\mu \mathrm{g} / \mathrm{kg} / \mathrm{min})\end{array}$ & 0.10 & $1(2.7 \%)$ & 0 \\
\hline$(0.09 / 0.13)$ & 0.11 & $6(16.2 \%)$ & 1 \\
\hline$(0.09 / 0.18)$ & 0.147 & $30(81 \%)$ & \\
\hline Average MAC & $\begin{array}{c}1.0 \\
(1.0 / 1.2)\end{array}$ & $\begin{array}{c}1.1 \\
(1.1 / 1.2)\end{array}$ & 0.068 \\
\hline $\begin{array}{l}\text { Intraoperative fluid administra- } \\
\text { tion (ml) }\end{array}$ & $598 \pm 198$ & $572 \pm 220$ & 0.646 \\
\hline Intraoperative MBP (mm Hg) & $61 \pm 4$ & $60 \pm 4$ & 0.770 \\
\hline Intraoperative HR (beats/min) & $62 \pm 6$ & $66 \pm 11$ & 0.064 \\
\hline Duration of anaesthesia (min) & $52 \pm 24$ & $50 \pm 30$ & 0.795 \\
\hline Blood loss (ml) & $75(0 / 188)$ & $\begin{array}{c}100 \\
(50 / 225)\end{array}$ & 0.029 \\
\hline NRS for surgical condition & $2(1 / 3)$ & $3(2 / 5)$ & 0.007 \\
\hline Boezaart score & $2(1 / 2)$ & $2(2 / 3)$ & 0.001 \\
\hline
\end{tabular}

The values are reported as the mean $\pm S D$ or median (1st/3rd quartiles). Group D, dexmedetomidine group; Group N, 0.9\% saline group; MAC, minimum alveolar concentration; MBP, mean blood pressure; $\mathrm{HR}$, heart rate; NRS, numeric rating scales; PACU, postanaesthesia care unit.

stoperative pain showed no difference between groups [1 (0/2) vs. $1(0 / 3), P=0.636]$. Three patients in group $D$ experienced dry mouth or throat postoperatively.

There was a correlation between LM score and blood loss in both groups $(r=0.546, P=0.004$ in group $D ; r=0.558, P=0.001$ in group $\mathrm{N}$ ). The $\mathrm{LM}$ score in group $\mathrm{N}$ showed a positive correlation with NRS $(r=0.421, P=0.021$, Figure $2 A)$ and Boezaart score $(r=0.476, P=0.008$, Figure $2 B$ ), whereas no significant correlation was observed between the LM score and NRS $(r=0.293, P$ $=0.164$, Figure $2 \mathrm{~A})$ or Boezaart score $(r=0.372, \mathrm{P}=0.061$, Figure 2B) in group $D$.

\section{Discussion}

Our results show that premedication with intranasal atomised DEX is associated with decreased bleeding and improved surgical conditions. Although patients receiving DEX stayed in the PACU longer, no increase in complications was observed. Intravenous DEX enabled safe, controlled hypotension and a
Table 3. Postoperative parameters.

\begin{tabular}{|c|c|c|c|}
\hline & $\begin{array}{l}\text { Group D } \\
(n=30)\end{array}$ & $\begin{array}{l}\text { Group N } \\
(n=30)\end{array}$ & P-value \\
\hline \multicolumn{4}{|l|}{ Recovery time } \\
\hline Extubation time (min) & $23 \pm 7$ & $21 \pm 6$ & 0.412 \\
\hline PACU time (min) & $35 \pm 8$ & $29 \pm 7$ & 0.007 \\
\hline \multicolumn{4}{|l|}{ Adverse events in PACU } \\
\hline Coughing & 1 & 0 & 1.000 \\
\hline Hypotension & 2 & 1 & 1.000 \\
\hline Hypoxemia & 0 & 1 & 1.000 \\
\hline Nausea & 1 & 0 & 1.000 \\
\hline \multicolumn{4}{|c|}{ Postoperative complications } \\
\hline Sore throat & 3 & 1 & 0.605 \\
\hline Dry mouth or throat & 3 & 0 & 0.236 \\
\hline VAS for pain intensity & $1(0 / 2)$ & $1(0 / 3)$ & 0.636 \\
\hline
\end{tabular}

Recovery time are reported as the mean \pm SD. VAS for postoperative pain is represented as the median ( $1 \mathrm{st} / 3$ rd quartiles). Other values are the number of patients. Group D, dexmedetomidine group; Group N, $0.9 \%$ saline group; PACU, postanaesthesia care unit; VAS, visual analogue scale.

dry surgical field for ESS ${ }^{(23)}$ that was superior to esmolol due to inherent analgesic, sedative, and anaesthetic-sparing effects ${ }^{(24)}$. Traditionally, controlled hypotension has been used to reduce the amount of bleeding and provide good intraoperative surgical conditions; however, many comparative studies demonstrated that hypotension on its own did not necessarily improve the surgical field ${ }^{(25)}$ and the amount of surgical bleeding was related to HR rather than blood pressure ${ }^{(18,26)}$. In our study, MBP was maintained at a deliberately hypotensive $55-65 \mathrm{mmHg}$ and HR did not differ significantly between groups. Meanwhile, the reverse Trendelenburg position, a proven factor contributing to surgical bleeding, was used in both groups. Thus, DEX administration was isolated as the experimental variable.

Furthermore, various DEX administration methods, such as intravenous and intranasal, have been used for anaesthetic management due to the sedative, analgesic, and anaesthetic-sparing effects. Intravenous DEX provides adequate sedation, haemodynamic stability, and better VAS scores and surgical comfort with fewer side effects for ESS patients receiving local anaesthesia $(27,28)$. Intranasal DEX administration was initially intended to provide sedation and pain relief for patients, and studies have shown that it is effective, convenient, and safe under local 
A

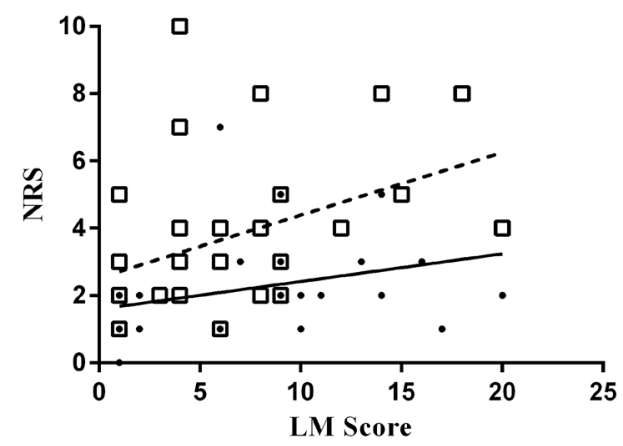

B

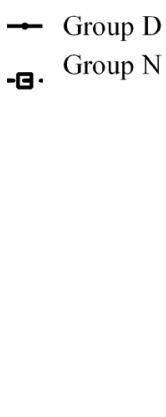

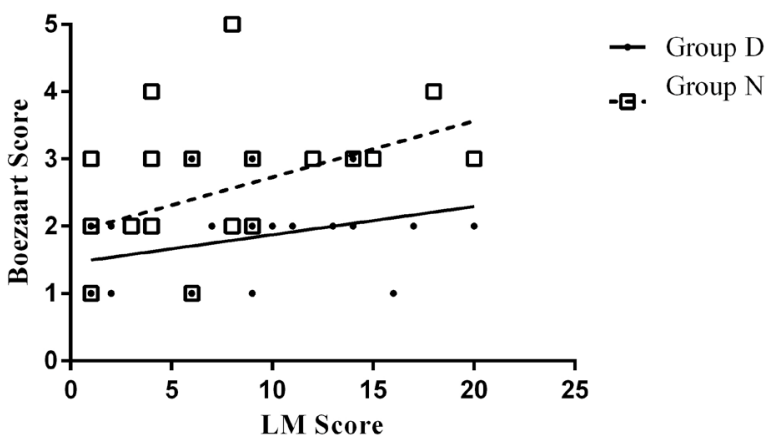

Figure 2. The correlation between LM score and variables for surgical condition (A, NRS; B, Boezaart score) in patients receiving intranasal dexmedetomidine (Group D) or $0.9 \%$ saline (Group N). LM score showed a positive correlation with NRS and Boezaat score in group N ( $\mathrm{P}=0.021$ and 0.008 , respectively), but not in Group D ( $\mathrm{P}=0.164$ and 0.061 , respectively). LM, Lund-Mackay; NRS, numeric rating scales.

anaesthesia ${ }^{(15,16)}$. Our results indicate that it possesses the additional advantages of reducing nasal bleeding and optimising the surgical field during ESS.

The mechanism for the favourable outcomes associated with DEX has not been fully clarified. The mechanism could be systemic, peripheral, or both. Anaesthetics modulate microcirculation primarily through autonomic sympathetic and parasympathetic nerves in vascular smooth muscle. Junctional a-1 and a-2 adrenoceptors coexist in the systemic vasculature, and both subtypes mediate vasoconstriction with $a-1$ adrenoceptors being preferentially innervated and $a-2$ adrenoceptors being primarily activated by circulating catecholamines (29). Intravenous DEX decreases oral mucosal blood flow ${ }^{(30)}$ and peripheral blood perfusion ${ }^{(31)}$ because the drug acts on peripheral $a-2$ adrenoceptors, inducing vasoconstriction ${ }^{(32)}$. Masuki et al. ${ }^{(33)}$ also suggested that DEX induces vasoconstriction via $a-2$ adrenoceptors in the human forearm. Specifically, vasoconstriction mediated by the direct activation of vascular $a-2$ adrenoceptors was attributed to the a-2A subtype, both in mice ${ }^{(34)}$ and humans ${ }^{(35)}$. Therefore, it is possible that DEX, an a-2 adrenoceptor agonist, improves surgical visibility via vasoconstriction. In addition to peripheral vasoconstriction, the nasal use of a-2 agonists during ESS can be valuable due to mixed, complicated actions when systemically absorbed. Kawaai et al. ${ }^{(30)}$ found that intravenous DEX resulted in a significant reduction in cardiac output caused by a reduction in HR, which could partially contribute to a reduction in oral mucosal blood flow. Our results showed slightly reduced $\mathrm{HR}$ with no significant difference in patients receiving intranasal DEX. Thus, the localised effects were assumed to play a dominant role in the action of intranasal DEX. As establishing intravenous access is painful and frightening for many patients, especially children, intranasal DEX premedication may be a feasible alternative to establishing venous access in anxious patients. DEX is rather rapidly and efficiently absorbed via a nasal atomisation device, which ensures proper volume delivery, enhances absorption, and improves bioavailability through the nose-brain pathway ${ }^{(36)}$. According to a previous study, following intranasal administration, the median time to reach peak plasma concentrations was $38 \mathrm{~min}$, and its absolute bioavailability was about $65 \%{ }^{(37)}$. Accounting for the induction time and the surgical instrument preparation time, we chose to deliver DEX 15 min prior to induction in our study to ensure peak DEX concentration during the surgery.

Although the PACU time in group D patients was prolonged for approximately $5 \mathrm{~min}$, surgical turnover may not be impaired in clinical institutions in which patients are resuscitated in the PACU instead of in the theatre room. Moreover, extubation time showed no differences between groups, which means that the extra 5 min was attributed to prolonged observation time after extubation in the PACU.

In our study, no improved pain relief was found $2 \mathrm{~h}$ postoperatively in patients administered nasal DEX, which was in accordance with previous studies that showed DEX to have a postoperative opioid-sparing effect but not clear pain relief (38) and no effect on pain pressure threshold ${ }^{(14)}$. Peripheral $a-2$ adrenoceptors themselves are not considered to have an analgesic effect in peripheral tissues ${ }^{(39)}$. As a result, DEX, even at a concentration of 10-6 M and without lidocaine, did not have any local anaesthetic effects ${ }^{(40)}$.

When interpreting the data presented in our study, some methodological aspects and limitations must be considered. The first is the reduced precision when measuring the amount of blood loss, i.e., the simple subtraction of the irrigation volume from the total volume collected in the canister ${ }^{(41)}$. Although an 
explorative attempt was made to improve the blood loss measurement by performing calculations based on the $\mathrm{Hb}$ values and the total volume collected in the canister ${ }^{(3)}$, determination of $\mathrm{Hb}$ values by arterial blood gas analyses is relatively invasive and resource consuming. Second, the lack of an objective method for surgical visibility assessment makes comparisons to previous studies difficult and may contribute to controversial results. As a novel approach, optical rhinometry was suggested to quantify sinonasal mucosal blood flow and correlate this level with blood loss and other clinical parameters ${ }^{(42)}$.

\section{Conclusions}

In conclusion, intranasal premedication with atomised $2 \mu \mathrm{g} / \mathrm{kg}$ DEX for ESS patients decreases the amount of blood loss from the surgical site and provides optimal surgical field visualisation.

\section{Acknowledgements}

The authors thank Dr. Dehui Wang, Yurong Gu and Limin Guo from Department of Otolaryngolory, The Eye Ear Nose and Throat Hospital, Fudan University, Shanghai, China for rating surgical visibility after surgery.

\section{Author contributions}

HQ: Manuscript preparation and data analysis; JC: Postoperative follow-up and data acquisition; WL: Guarantor of integrity of entire study. XS: Conception and design of the study and manuscript revision.

\section{Conflict of interest}

No external funding and no competing interests declared.

\section{References}

1. Stankiewicz JA, Lal D, Connor M, Welch K. Complications in endoscopic sinus surgery for chronic rhinosinusitis: A 25-year experience. Laryngoscope 2011;121:2684-2701.

2. Drozdowski A, Sieskiewicz A, Siemiatkowski A. [Reduction of intraoperative bleeding during functional endoscopic sinus surgery]. Anestezjol Intens Ter 2011;43:45-50.

3. Ahn HJ, Chung SK, Dhong HJ, et al. Comparison of surgical conditions during propofol or sevoflurane anaesthesia for endoscopic sinus surgery. Br J Anaesth 2008; 100:50-54

4. Khosla AJ, Pernas FG, Maeso PA. Metaanalysis and literature review of techniques to achieve hemostasis in endoscopic sinus surgery. Int Forum Allergy Rhinol 2013;3:482-487

5. Mohseni M, Ebneshahidi A. The effect of oral clonidine premedication on blood loss and the quality of the surgical field during endoscopic sinus surgery: A placebo-controlled clinical trial. J Anesth 2011;25:614617.

6. Wawrzyniak K, Kusza K, Cywinski JB, Burduk PK, Kazmierczak W. Premedication with clonidine before TIVA optimizes surgical field visualization and shortens duration of endoscopic sinus surgery - results of a clinical trial. Rhinology 2013;51:259-264.

7. Wawrzyniak K, Burduk PK, Cywinski JB, Kusza K, Kazmierczak W. Improved quality of surgical field during endoscopic sinus surgery after clonidine premedication--a pilot study. Int Forum Allergy Rhinol 2014;4:542547.

8. Gabriel JS, Gordin V. Alpha 2 agonists in regional anesthesia and analgesia. Curr Opin Anaesthesiol 2001;14:751-753.

9. Hamasaki J, Tsuneyoshi I, Katai R, Hidaka T, Boyle WA, Kanmura Y. Dual alpha(2)-adrenergic agonist and alpha(1)-adrenergic antagonist actions of dexmedetomidine on human isolated endothelium-denuded gastroepiploic arteries. Anesth Analg
2002;94:1434-1440.

10. Duma A, Urbanek B, Sitzwohl C, Kreiger A, Zimpfer M, Kapral S. Clonidine as an adjuvant to local anaesthetic axillary brachial plexus block: A randomized, controlled study. Br J Anaesth 2005;94:112-116.

11. Kanazi GE, Aouad MT, Jabbour-Khoury SI, et al. Effect of low-dose dexmedetomidine or clonidine on the characteristics of bupivacaine spinal block. Acta Anaesthesiol Scand 2006;50:222-227.

12. Esmaoglu A, Yegenoglu F, Akin A, Turk CY. Dexmedetomidine added to levobupivacaine prolongs axillary brachial plexus block. Anesth Analg 2010;111:1548-1551.

13. Brummett CM, Hong EK, Janda AM, Amodeo FS, Lydic R. Perineural dexmedetomidine added to ropivacaine for sciatic nerve block in rats prolongs the duration of analgesia by blocking the hyperpolarization-activated cation current. Anesthesiology 2011;115:836-843

14. Yuen VM, Irwin MG, Hui TW, Yuen MK, Lee LH. A double-blind, crossover assessment of the sedative and analgesic effects of intranasal dexmedetomidine. Anesth Analg 2007;105:374-380.

15. Nooh N, Sheta SA, Abdullah WA, Abdelhalim AA. Intranasal atomized dexmedetomidine for sedation during third molar extraction. Int J Oral Maxillofac Surg 2013;42:857-862.

16. Cheung CW, Ng KF, Liu J, Yuen MY, Ho MH, Irwin MG. Analgesic and sedative effects of intranasal dexmedetomidine in third molar surgery under local anaesthesia. $\mathrm{Br} J$ Anaesth 2011;107:430-437.

17. Lund VJ, Mackay IS. Staging in rhinosinusitus. Rhinology 1993;31:183-184.

18. Eberhart LH, Folz BJ, Wulf H, Geldner G. Intravenous anesthesia provides optimal surgical conditions during microscopic and endoscopic sinus surgery. Laryngoscope 2003;113:1369-1373.

19. Jacobi KE, Bohm BE, Rickauer AJ, Jacobi C, Hemmerling TM. Moderate controlled hypotension with sodium nitroprusside does not improve surgical conditions or decrease blood loss in endoscopic sinus surgery. J Clin Anesth 2000;12:202-207.

20. Boezaart AP, van der Merwe J, Coetzee A. Comparison of sodium nitroprusside- and esmolol-induced controlled hypotension for functional endoscopic sinus surgery. Can J Anaesth 1995;42:373-376.

21. Ragab SM, Hassanin MZ. Optimizing the surgical field in pediatric functional endoscopic sinus surgery: A new evidencebased approach. Otolaryngol Head Neck Surg 2010;142:48-54.

22. Cho K, Lee JY, Park SK, et al. Comparison of surgical conditions during propofol or desflurane anesthesia for endoscopic sinus surgery. Korean J Anesthesiol 2012;63:302-307.

23. Lee J, Kim Y, Park C, et al. Comparison between dexmedetomidine and remifentanil for controlled hypotension and recovery in endoscopic sinus surgery. Ann Otol Rhinol Laryngol 2013;122:421-426.

24. Shams T, El BN, Abu-Samra M, El-Masry R. Induced hypotension for functional endoscopic sinus surgery: A comparative study of dexmedetomidine versus esmolol. Saudi J Anaesth 2013;7:175-180.

25. Jacobi KE, Bohm BE, Rickauer AJ, Jacobi C, Hemmerling TM. Moderate controlled hypotension with sodium nitroprusside does not improve surgical conditions or decrease blood loss in endoscopic sinus surgery. J Clin Anesth 2000;12:202-207.

26. Nair S, Collins M, Hung P, Rees G, Close D, Wormald PJ. The effect of beta-blocker premedication on the surgical field during endoscopic sinus surgery. Laryngoscope 2004; 114:1042-1046.

27. Goksu S, Arik H, Demiryurek S, Mumbuc S, Oner U, Demiryurek AT. Effects of dexmedetomidine infusion in patients undergoing functional endoscopic sinus surgery under local anaesthesia. Eur J Anaesthesiol 2008;25:22-28.

28. Guven DG, Demiraran Y, Sezen G, Kepek 
O, Iskender A. Evaluation of outcomes in patients given dexmedetomidine in functional endoscopic sinus surgery. Ann Otol Rhinol Laryngol 2011;120:586-592.

29. Langer SZ, Shepperson NB, Massingham R. Preferential noradrenergic innervation of alpha-adrenergic receptors in vascular smooth muscle. Hypertension 1981;3:1112-1118.

30. Kawaai H, Yoshida K, Tanaka E, et al Dexmedetomidine decreases the oral mucosal blood flow. Br J Oral Maxillofac Surg 2013;51:928-931.

31. Gargiulo S, Gramanzini M, Liuzzi R, Greco A, Brunetti A, Vesce $G$. Effects of some anesthetic agents on skin microcirculation evaluated by laser Doppler perfusion imaging in mice. BMC Vet Res 2013;9:255.

32. Koss MC. Differential neural activation of vascular alpha-adrenoceptors in oral tissues of cats. Eur J Pharmacol 2002;440:53-59.

33. Masuki S, Dinenno FA, Joyner MJ, Eisenach $\mathrm{JH}$. Selective alpha2-adrenergic properties of dexmedetomidine over clonidine in the human forearm. J Appl Physiol (1985) 2005;99:587-592.

34. Duka I, Gavras I, Johns C, Handy DE, Gavras H. Role of the postsynaptic alpha(2)-adren- ergic receptor subtypes in catecholamineinduced vasoconstriction. Gen Pharmacol 2000;34:101-106.

35. Philipp M, Brede M, Hein L. Physiological significance of alpha(2)-adrenergic receptor subtype diversity: One receptor is not enough. Am J Physiol Regul Integr Comp Physiol 2002;283:R287-R295.

36. Merkus P, Ebbens FA, Muller B, Fokkens WJ. The 'best method' of topical nasal drug delivery: Comparison of seven techniques. Rhinology 2006;44:102-107.

37. Iirola T, Vilo S, Manner T, et al. Bioavailability of dexmedetomidine after intranasal administration. Eur J Clin Pharmacol 2011;67:825-831.

38. Chan AK, Cheung CW, Chong YK. Alpha-2 agonists in acute pain management. Expert Opin Pharmacother 2010;11:2849-2868.

39. MacDonald E, Kobilka BK, Scheinin M. Gene targeting--homing in on alpha 2-adrenoceptor-subtype function. Trends Pharmacol Sci 1997;18:211-219.

40. Yoshitomi T, Kohjitani A, Maeda S, Higuchi H, Shimada M, Miyawaki T. Dexmedetomidine enhances the local anesthetic action of lidocaine via an alpha-2A adrenoceptor. Anesth Analg
2008;107:96-101.

41. Manola M, De Luca E, Moscillo L, Mastella A. Using remifentanil and sufentanil in functional endoscopic sinus surgery to improve surgical conditions. ORL J Otorhinolaryngol Relat Spec 2005;67:83-86.

42. Gomez-Rivera F, Cattano D, Ramaswamy $U$, et al. Pilot study comparing total intravenous anesthesia to inhalational anesthesia in endoscopic sinus surgery: Novel approach of blood flow quantification. Ann Otol Rhinol Laryngol 2012;121:725-732.

Xia Shen

Department of Anesthesiology

The Eye Ear Nose and Throat Hospital

Fudan University

83 Fenyang Road

Shanghai 200031

China

E-mail:shenxiash@gmail.com 